\title{
Les femmes au Grand Siècle. Le Baroque: musique et littérature. Musique et liturgie. Edités par David Wetsel et Frédéric Canovas
}

\section{Letizia Norci Cagiano}

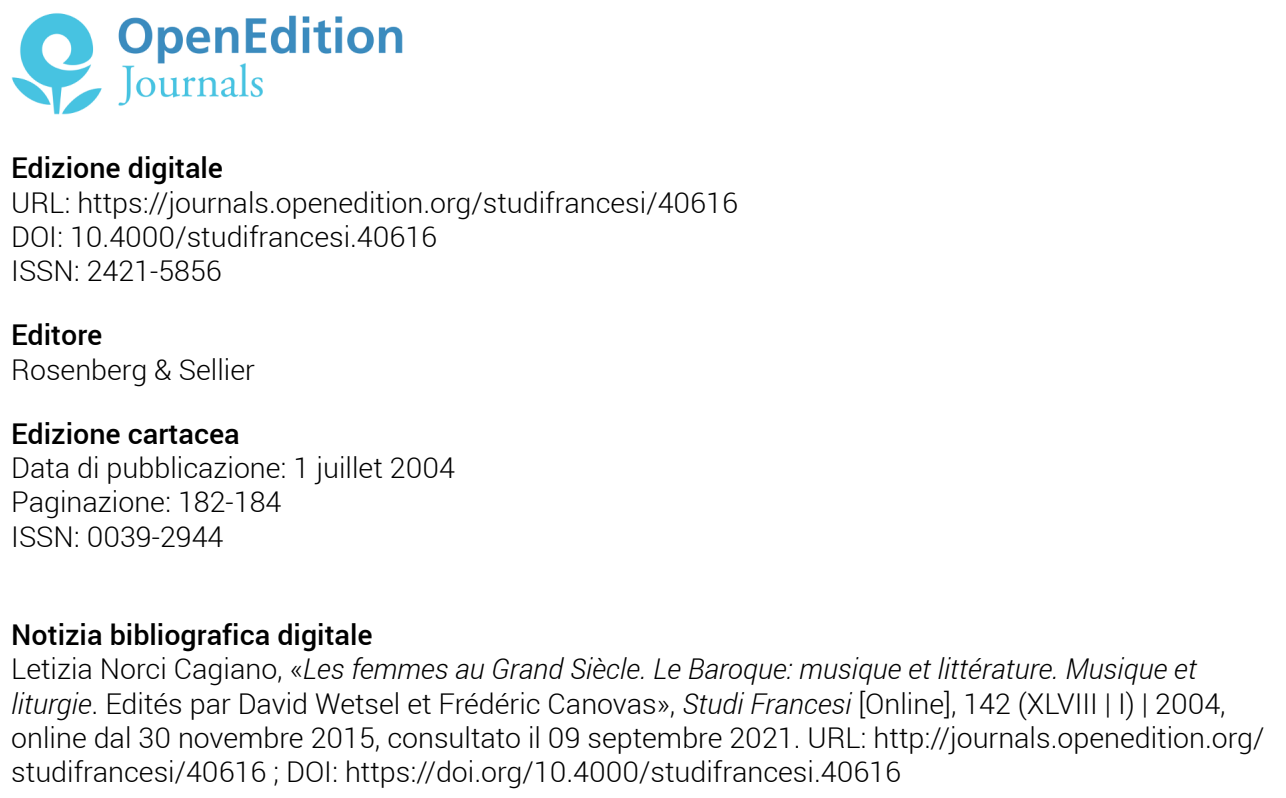

Questo documento è stato generato automaticamente il 9 septembre 2021.

\section{(ब) $\odot \Theta \Theta$}

Studi Francesi è distribuita con Licenza Creative Commons Attribuzione - Non commerciale - Non opere derivate 4.0 Internazionale. 


\title{
Les femmes au Grand Siècle. Le Baroque: musique et littérature. Musique et liturgie. Edités par David Wetsel et Frédéric Canovas
}

\author{
Letizia Norci Cagiano
}

\section{NOTIZIA}

Les femmes au Grand Siècle. Le Baroque: musique et littérature. Musique et liturgie. Actes du $33^{\circ}$ congrès annuel de la North American Society for Seventeenth-Centwy French Literature, Tome II, Arizona State University (Tempe), May 2001, Edités par David Wetsel et Frédéric Canovas, Tübingen, Gunter Narr Verlag, 2003, «Biblio 17», pp. 269.

1 Questo volume raccoglie gli atti di un convegno dedicato quasi esclusivamente a figure femminili del Grand Siècle (16 saggi) e in minima parte alla musica barocca (2 saggi su musica e letteratura e uno su musica e liturgia), con un'appendice di John F. Boitano dal titolo Paris of the 'Ancien Régime': An Interdisciplinary Course on Cities and Civilizations.

Gli organizzatori del convegno hanno voluto ricollegarsi agli studi sulle donne in epoca classica già raccolti da Wolfgang Leiner nella collezione delle Études littéraires françaises (1978 e 1984). Dall'epoca della prima pubblicazione di Leiner, le ricerche su questa tematica si sono moltiplicate in Europa e in altre parti del mondo, sull'onda del dilagante interesse per la questione fermminile. Gli autori di questi saggi hanno quindi potuto consultare e mettere felicemente a frutto una ricca bibliografia per scavare nella vita o nelle opere di donne celebri (Angélique Arnauld, Cristina di Svezia, Madame Guyon, Caterina Biancolelli), per scoprire lati inediti di personaggi letterari (in particolare le protagoniste del teatro di Molière, ma anche di Marie-Anne Barbier, di Scarron, di Racine e della poco nota suora De la Chapelle, autrice di una tragedia su Santa Caterina d'Alessandria) o per trattare questioni più generali, come il preziosismo, 
la galanteria, l'eroismo, il ruolo letterario della donna, senza contare altri saggi di argomenti svariati riguardanti il romanzo d'avventura e la narrativa fiabesca.

Abbiamo dunque un vasto panorama il cui pregio (ed eventuale limite) consiste, tra l'altro, nell'angolazione da cui viene osservato il mondo francese del Grand Siècle. L'impressione che danno questi (come tanti altri) studi americani è la stessa di quando si osserva un luogo famigliare da un punto di vista insolito: il luogo è sempre quello, eppure ci appare estraneo, bizzarro, privato di quell'armonia che lo sguardo costruisce attraverso l'abitudine e che noi crediamo scontata ed immutabile. Si scorgono particolari nuovi, le distanze e le proporzioni appaiono sovvertite, alcuni oggetti difficili da individuare si rivelano in piena luce, mentre altri scompaiono. Emergono dettagli che prima, per la loro eccessiva evidenza, non erano neppure presi in considerazione; oppure si manifestano curiosità e meraviglia nei confronti di comportamenti normali per la nostra tradizione di europei.

Il saggio di Marie-Odile Sweetser, Voix féminines dans la littérature classique, può essere citato come esempio di questa diversità di prospettive. Un primo senso di perplessità è dato dall'accostamento delle voci che, seconda una logica tradizionale, appaiono eterogenee se non addirittura incompatibili: la poetessa Marie-Catherine Desjardins, l'Agnès di Molière, l'Andromaque di Racine, la princesse de Clèves e Madame de Maintenon. Difficilmente un europeo avrebbe potuto concepire un simile cocktail di personaggi reali ed immaginari, espressione di caratteri e di tendenze culturali tanto diverse tra loro; e tuttavia la Sweetser riesce ad individuare un'istanza comune: la ricerca di un'autonomia femminile e il tentativo di realizzare in questo senso, almeno nella sfera privata, le promesse dell'Umanesimo. Si giustifica così l'accostamento di Marie-Catherine Desjardins, poetessa capace di esprimere la passione con la stessa libertà dei suoi «confrères masculins», con l'ingenua Agnès cui l'amore apre gli occhi, l'affettività e l'intelligenza inducendola a rivendicare l'educazione che non ha avuto. Viene tracciato in questo modo un percorso della coscienza che trova il suo caposaldo in Andromaque, simbolo della fedeltà a se stessa e ai valori in cui crede: in mezzo alle sventure l'eroina di Racine è cosciente della sua missione (assicurare la continuità della dinastia di Ettore) e pronta a morire per difendere i suoi sentimenti più cari, la sua identità e l'integrità della sua persona. Anche la princesse de Clèves pone la questione di una conoscenza di sé svincolata dagli schemi forniti dalla società. Questa conoscenza si afferma ripetutamente, nel romanzo, attraverso la «prise de parole» della protagonista: nella scena famosa dell'«aveu» (e qui la Sweetser fa riferimento all'analisi di Jean Garapon) e in quella dell'ultimo incontro con Nemours, in cui le decisioni prese in totale autonomia vengono affermate irrevocabilmente.

Dopo aver accennato di sfuggita anche alla figura di Madame de Maintenon, la Sweetser conclude osservando, con una certa meraviglia, che, «en dépit des structures existantes et persistantes d'une société patriarcale, renforcées par l'ordre d'une monarchie absolue, en dépit des mentalités prévalentes, combattues par des groupes mondains et savants, des écrivains ont pu concevoir des personnages de femmes autonomes et leur donner une voix» (p. 52).

6 Tale meraviglia, che percorre questo ed altri saggi della raccolta, mi sembra appartenere anch'essa al panorama visto d'oltreoceano, legata com'è ad una propensione a proiettare il femminismo (come altre questioni o punti di vista oggi) su una cultura che non poneva la questione in termini generali, lasciando piuttosto molta libertà alla donna di affermare se stessa in modo individuale, coi suoi propri mezzi, in 
un rapporto con l'uomo che si pone a livello singolo e il cui equilibrio è lasciato alle risorse del carattere, dell'intelligenza, dell'istinto, della femminilità. Tanto è vero che gli esempi portati dalla Sweetser sono eterogenei e l'unico filo che li collega è quello delle risorse intime ed originali dei singoli personaggi.

$7 \mathrm{Ci}$ potremmo chiedere a questo punto quanto sia giusto leggere in chiave di femminismo moderno le gesta delle nostre antenate d'Ancien Régime e fino a che punto sia stimolante applicare - con maggiore o minore spregiudicatezza - $\mathrm{i}$ canoni del terzo millennio all'interpretazione del passato. È più raccomandabile osservare dall'esterno o esplorare, nei limiti del possibile, dall'interno, attraverso un'immedesimazione che ricorre, oltre alle conoscenze storiche, artistiche e letterarie, anche alla tradizione e all'ereditarietà? Tutti siamo affezionati al punto di vista della tradizione: un albero piantato dai nostri nonni, che abbiamo visto crescere, di cui ogni giorno osserviamo la scorza rugosa, che magari abbiarno descritto o disegnato, che abbiamo contemplato e fatto partecipe delle nostre emozioni, la cui immagine è stampata nella memoria, la cui impressione affiora dal nostro subconscio, siamo portati a considerarlo in modo diverso dagli altri alberi e a caricarlo di significati che corrispondono ai nostri valori e a quelli della nostra cultura. Diciamo che siamo compiaciuti di vederlo così, di contemplarne l'immagine nel nostro caleidoscopio. Ma ecco che arriva qualcuno dal di fuori e dà un colpetto al caleidoscopio: il mosaico è sconvolto, i rapporti cambiano, abbiamo una configurazione nuova ed estranea che ha qualcosa di famigliare, ma in cui non ci ritroviamo più. È un intervento che non ci sembra di avere autorizzato, che ci costringe a rimettere tutto in questione, a rivedere, a riconsiderare, anche a domandarci se dobbiamo adeguarci al nuovo punto di vista o indurre gli altri ad adottare il nostro.

Benvenga dunque questo intervento, stimolante e certamente produttivo, a patto però che non induca all'adeguamento, che sia mantenuta una dialettica tra le culture, che lo sguardo esterno rimanga tale e non si cada nella tentazione di farlo nostro, di generalizzare e di livellare. Con queste avvertenze l'applicazione dei modelli globali di oggi al mondo di Luigi XIV diventa un esperimento utile per mettere in risalto aspetti oscuri o poco considerati di quell'epoca o anche per scoprire lati insospettati di personaggi noti; ed è quanto avviene per alcuni dei saggi di questa raccolta che attraggono proprio per l'originalità degli aspetti che emergono da un approccio non si sa bene se ingenuo, disinvolto o volutamente in contrasto con i canoni della critica europea.

9 Spigolando tra tante figure e immagini, tra analisi raffinate e originali del mondo femminile del Grand Siècle, mi vorrei ancora soffermare sul personaggio di Madame Guyon, così come lo presenta Mary M. Randall nel suo saggio Mystic Edge or Mystic on the Edge? Madame Guyon Revisited. Dopo aver considerato gli atteggiamenti contrastanti (anche da parte della Chiesa) che si sono rnanifestati attraverso i tempi nei confronti dei mistici, la Randall analizza la vicenda del quietismo come uno degli episodi del declino del misticismo europeo, del crépuscule des mystiques, per usare un'espressione di Louis Cognet.

10 L'eperienza religiosa di Madame Guyon è presentata come un dramma teatrale di cui Luigi XIV sarebbe stato il regista e i principali protagonisti Bossuet, campione della chiesa Gallicana e Fénelon, l'ultramontano, difensore degl'interessi del Vaticano. O meglio ancora, per restare nell'ambito dei gusti teatrali del XVII secolo, come un balletto, "a theological dance», di cui ogni passo è studiato con estrema cautela: «Bossuet is dancing to Madame de Maintenon's tune. His movements are robust, 
blustering, grandiose. Fénelon moves with grace, precision, refinement. They circle round and round. The locus of their maneuverings is the mystic, Jeanne-Marie Bouvier de la Mothe Guyon, better known as Madame Guyon» (p. 109).

11 Mme Guyon e il suo ruolo controverso nella Querelle du quiétisme sono quindi oggetto di una serie d'indagini che trasformano progressivamente il dramma abbozzato all'inizio di questo saggio in un vero e proprio processo: la Randall cerca infatti di dimostrare che la signora in questione fu imprigionata per otto anni e sottoposta a logoranti pressioni psicologiche senza che vi fosse in realtà alcuna prova a sua carico. I motivi che portarono all'ingiusta condanna, elencati da 1 a 10 , sono di natura molto varia: a partire dalle questioni politiche, religiose e morali (e tra queste le «constraints imposed on women»), fino a quella che Mary Randall definisce "a preoccupation with the 'self"»: "The seventeenth-cmtury focus on the self - spiega - impacted Madame Guyon's reception in her own time. Reciprocally, her own self-image and the images of her that emerge from renewed focus on her life and works offer us better understanding of ourselves now» (p. 111).

Partendo da quest'ultima considerazione, Mary Randall cerca di esplorare la figura di Madame Guyon sulla scorta degli studi di Ronald Knox e di Michel de Certeau, ma soprattutto attraverso la lettura delle sue opere autobiografiche (i Récits de captivité e La vie écrite par elle-même). L'analisi si sofferma in particolare sull'immagine dell'acqua: «She, like pure water, with no characteristic shape or colour of its own, is fluid and extensive».

Rien de plus simple que l'eau, - prosegue citando La vie écrite par elle-même - rien de plus pur; mais cette eau a une étendue admirable à cause de sa fluidité. Elle a aussi qualité, qui n'ayant nulle qualité propre elle prend toutes sortes d'impressions. Elle n'a nul goût et elle prend tous les goûts: elle n'a nulle couleur, et elle prend toutes les couleurs.

tetando se stessa di ogni qualità propria, Madame Guyon, come l'acqua, suscita una miriade d'immagini nella fantasia altrui, ponendosi come un "provocative subject». Infatti, osserva Mary Randall, Madame Guyon ha una ben precisa immagine di sé, come prova il fatto che abbia scritto un'autobiografia, operazione che comporta «observance, report, and commentary of her own life».

Potrebbe essere interessante ricollegarsi, a questo proposito, alle immagini dell'acqua nell'opera di Fénelon e alle interpretazioni che ne ha dato François-Xavier Cuche; ma questo saggio si concentra sulla figura e sulla difesa della protagonista e, come l'altro che abbiamo analizzato, insiste sulla presa di coscienza di un personaggio femminile del XVII secolo. Presa di coscienza che resta il punto fermo di una figura che si è prestata alle interpretazioni più diverse, a critiche violente nel periodo della Querelle, a un lungo oblio e, finalmente, negli ultimi tempi, ad una rivalutazione, a prova della lungimiranza di una sua celebre frase: «Plus les choses sont simples et pures, plus elles ont de l'étendue».

Dopo tante pagine dedicate alle donne del XVII secolo, meraviglia lo spazio esiguo riservato alla seconda e terza parte di questo volume. In realtà i tre saggi finali vogliono solamente illustrare la Messa di Requiem di Jean Gilles, celebrata durante il convegno in memoria di James Anthony, uno dei più quotati studiosi americani di musica barocca. Solo uno dei tre studi, l'ultimo, di Patricia Ranum, è dedicato esclusivamente a Jean Gilles, al rapporto tra musica e letteratura sacra e profana e in particolare alla retorica utilizzata dal compositore per suscitare le emozioni degli ascoltatori. Il tema delle interferenze tra musica liturgica e musica teatrale è ripreso nell'analisi di Margot 
Martin sulla Médée di Charpentier. Più ampio è invece il tema trattato da Perry Gethner che, analizzando il rapporto tra musica e follia, ha modo di estendersi anche sul problema della combinazione di recitazione teatrale e musica nelle rappresentazioni seicentesche.

17 Si aggiunge così un breve, ma interessante contributo alla rinascita d'interesse per la musica sei-settecentesca e per la sua riproduzione filologica; interesse vivo già da molti anni in Francia, e ormai sentito anche in Italia, come provano l'attività del Centro di Studi sul Rinascirnento Musicale ad Artimino e del Conservatorio della Pietà dei Turchini a Napoli o anche i convegni scientifici come quello dedicato a Lully, l'opera e la parodia dell'opera (Roma, febbraio 2000) o l'altro, recentissimo, organizzato a Villa Medici da Patrick Dandrey e Nella Anfuso su Musica, letteratura e viaggiatori francesi nella Roma dei XVII secolo (settembre 2003). 